t's a Tuesday morning at $11.30 \mathrm{am}$. You are already 45 minutes behind. A 35 year old woman is referred to your neurology clinic with a nine month history of fatigue, dizziness, back pain, left sided weakness, and reduced mobility. Her general practitioner documents a hysterectomy at the age of 25, subsequent division of adhesions for abdominal pain, irritable bowel syndrome, and asthma. She is no longer able to work as a care assistant and rarely leaves the house. Her GP has found some asymmetrical weakness in her legs and wonders if she may have developed multiple sclerosis. She looks unhappy but becomes angry when you ask her whether she is depressed. On examination you note intermittency of effort and clear inconsistency between her ability to walk and examination on the bed. She has already had extensive normal investigations. The patient and her husband want you to "do something". As you start explaining that there's no evidence of anything serious and that you think it's a psychological problem, the consultation goes from bad to worse....

In this article we summarise an approach to the assessment and diagnosis of functional symptoms in neurology, paying attention to those symptoms that are particularly "neurological", such as paralysis and epileptic-like attacks. In the second of the two articles we describe our approach to the management of functional symptoms bearing in mind the time constraints experienced by a typical neurologist. We also address difficult questions such as: "What causes functional symptoms?", "Are they real?", and "Is there anything that can be done?"

We emphasise the need for a transparent and collaborative approach. As we will explain this depends on giving up a purely "psychological" view of functional symptoms in favour of a biopsychosocial view of causation in which dysfunction of the nervous system is the final common pathway.

\section{SYMPTOMS AND DISEASE}

It is important to keep in mind the difference between symptoms and disease. Symptoms, like fatigue, are the patient's subjective experience. Doctors are trained to find a disease, such as multiple sclerosis, to explain the symptoms. When there is no disease it becomes tempting to suggest that the symptom must be "not real" or psychogenic. In fact, symptoms appear for multiple reasons of which disease is only one (fig 1). Symptoms arise from physiological factors (for example, physiological tremor), psychological factors (for example, paraesthesia during a panic attack), behaviours (for example, excessive rest), and cultural or external factors (for example, compensation and the welfare state). For some patients disease pathology is a major (but not the only) factor in causing symptoms and in others it is minor or absent entirely.

A crucial implication of this approach is that the patient does not have to have a "genuine" disease in order to have a "genuine" symptom.

\section{WHAT SHOULD WE CALL THEM?}

The large number of terms to describe symptoms unexplained by disease is a reflection of the diverse concepts that have been used to understand them. They include:

- Pure symptomatic labels (for example, chronic fatigue, low back pain)

- Symptom syndromes (for example, chronic fatigue syndrome)

- "Non-diagnoses" that describe what the diagnosis is not rather than what it is (for example, non-epileptic attacks, non-organic, medically unexplained)

See end of article for authors' affiliations

Correspondence to: Dr Jon Stone, Department of Clinical Neurosciences, Western General Hospital, Crewe Road, Edinburgh EH4 2XU, UK; Jon.Stone@ed.ac.uk
- Diagnoses that imply an as yet unestablished disease cause (for example, reflex sympathetic dystrophy)

- Diagnoses that imply an as yet unestablished psychological cause (psychogenic, psychosomatic, "all in your mind")

- Historic diagnoses that do not fit in to any of these categories (for example, "hysteria", "functional")

- "Official" psychiatric diagnoses. These are found in psychiatric glossaries which are rarely used by neurologists and include: 


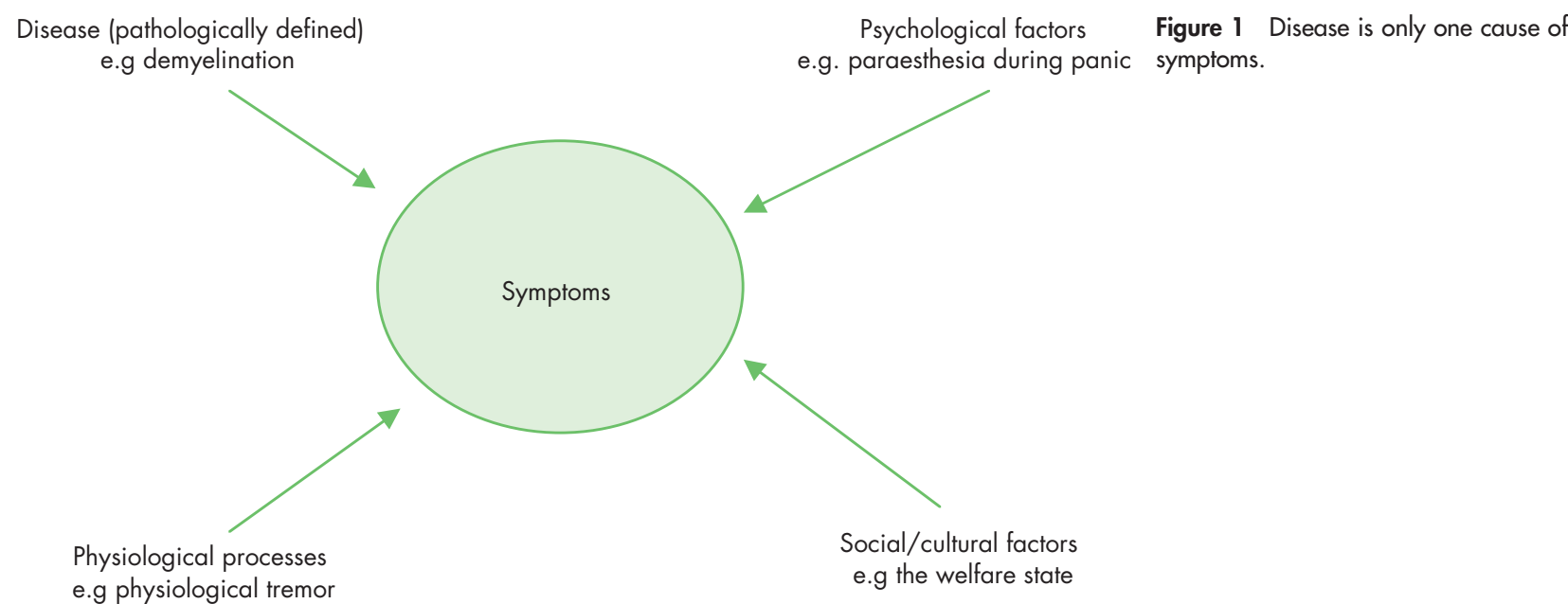

- conversion disorder: a psychoanalytic concept that describes the occurrence of motor or sensory neurological symptoms other than pain and fatigue that cause distress, are not explained by disease, not malingered but are thought to relate to psychological factors

- somatisation disorder (Briquet's syndrome): refers to patients with lifelong functional symptoms including pain, neurological, gastrointestinal and sexual symptoms, again with the implication that psychological problems have been somatised or converted

- dissociative motor disorder: in which dissociation (or a failure of integration of psychological processes) is the putative mechanism

- hypochondriasis: a distressing state of anxiety about disease

- factitious disorder: symptoms consciously simulated in order to gain medical care

- malingering: a term (and not a medical diagnosis) for symptoms which are simulated for clear financial or material gain.

The terminology you use is important. It will not only reflect how you think about the problem, but will also determine the patients' reaction to your diagnosis. For reasons that we explain in the second article we prefer the term "functional" and will use it in these articles.

\section{HOW COMMON ARE FUNCTIONAL SYMPTOMS?}

Around one third of new neurological outpatients have symptoms regarded by neurologists as "not at all" or only "somewhat" explained by disease. ${ }^{1}$ This finding is not unique to neurology and has also been reported in primary and secondary medical care worldwide. Table 1 illustrates some of the different functional somatic symptoms and syndromes that have been described by various medical specialties. Although superficially disparate, there is substantive overlap in the symptoms, epidemiology, and response to treatments of these functional somatic syndromes. ${ }^{2}$

Contrary to popular belief, even the more dramatic functional symptoms are surprisingly common. The incidence of functional paralysis is probably similar to that of multiple sclerosis (around 5/100 000). Non-epileptic attacks make up around $10-20 \%$ of the patients referred to specialist epilepsy clinics with intractable seizures and up to $50 \%$ of patients admitted to hospital in apparent status epilepticus. ${ }^{3}$ In movement disorders clinics up to $5 \%$ of new referrals may have functional symptoms. ${ }^{4}$

\section{FUNCTIONAL SYMPTOMS: WHY BOTHER?}

When faced with a clinic full of patients with epilepsy and multiple sclerosis, many neurologists cannot help thinking that patients with functional symptoms should be at the bottom of their priorities. There are a variety of views. Many doctors believe that patients often exaggerate or make up their symptoms in order to gain sympathy or financial benefit. Alternatively, some doctors believe the patients' symptoms, but simply view the problem as "not neurological" and one that should be dealt with by a psychiatrist and not a neurologist.

One argument relates to the patients themselves. When patients with functional neurological symptoms are compared to those whose symptoms are associated with disease, they are found to have similar disability and even more distress. ${ }^{1}$ Their symptoms tend to persist at follow up but only rarely become explained by disease. Distress and disability are by their nature subjective but we argue that ultimately it is the subjective that matters most.

The second argument relates to the work of a neurologist. Whether you like it or not, functional symptoms account for one third of your workload. If you allow yourself to become interested in the problem rather than irritated by it, you may find it has an effect on how much you enjoy your job generally.

Table 1 Examples of "functional" somatic symptoms and syndromes from different medical specialities

\begin{tabular}{ll} 
Neurology & $\begin{array}{l}\text { Functional weakness, non-epileptic attacks, } \\
\text { hemisensory symptoms }\end{array}$ \\
Gastroenterology & $\begin{array}{l}\text { Irritable bowel syndrome, non-ulcer } \\
\text { dyspepsia, chronic abdominal pain } \\
\text { Chronic pelvic pain, premenstrual syndrome }\end{array}$ \\
Gynaecology & $\begin{array}{l}\text { Functional dysphonia, globus pharynges } \\
\text { ENT }\end{array}$ \\
Cardiology & Atypical chest pain, unexplained palpitations \\
Rheumatology & Fibromyalgia \\
Infectious diseases & (Post-viral) chronic fatigue syndrome \\
\hline Immunology/allergy & Multiple chemical sensitivity syndrome \\
\hline
\end{tabular}




\section{TAKING A HISTORY FROM SOMEONE WITH FUNCTIONAL SYMPTOMS: A PRACTICAL APPROACH}

If you suspect that a patient's presenting symptoms are functional, there are ways of adapting the history to make it more efficient, more interesting for you, and more helpful for the patient. For the patient with functional symptoms, a good assessment is also the beginning of treatment.

\section{"Drain the symptoms dry"}

If your patient has a lot of symptoms, begin by making a list of all of them. Here is where you can save most time by resisting the urge to interrogate the features and onset of every symptom as you go. Instead, leave a few lines between each symptom on the list so that you can return to them as required. This allows the patient to unburden themselves quickly of all their symptoms, gives you a broad picture early on, and prevents new symptoms "cropping up" late in the consultation. Fatigue, sleep disturbance, memory and concentration problems, and pain can be routinely enquired about at this stage. However, for reasons we will explain, questions about mood are often better left to later.

The more physical symptoms a patient presents with the more likely it is that the primary presenting symptom will not be explained by disease. ${ }^{2}$ A long list of symptoms should therefore be a "red flag" that the main symptom is functional.

\section{Asking about disability}

Ask the patient to describe "What's a typical day like?". Follow up questions such as "How much of the day do you spend in bed?" and "How often do you leave the house?" are more useful than the traditional disability questions about dressing and walking distance. Pay particular attention to why they are disabled-for example, someone may have a very mild hemiparesis which really does not impair gait but be very worried about falling which is why they do not go outside.

\section{Finding out more about onset and course}

Although you may want to take a detailed history of the course of some symptoms, if a patient has had the symptoms for many years it may be more useful to obtain the overall course of the illness by drawing a graph with time on the $\mathrm{x}$ axis and severity on the y axis (fig 2). This can be a quick way of condensing a large amount of information - the line of the graph demonstrates how the illness has gradually worsened, cycled, or perhaps just been static over the period in question. To find the starting point, a useful question is "When did you last feel well?". Other events can then be added using arrows-for example, to indicate when the patient stopped working, life events, or medical interventions.

\section{Asking about dissociation}

Dissociative symptoms include depersonalisation (feeling detached from oneself) and derealisation (feeling that the world is no longer real) and can be unfamiliar territory for neurologists. However, they commonly occur in patients with neurological disease (such as epilepsy and migraine), in patients with functional symptoms, particularly those with paralysis and non-epileptic attacks, and less commonly in healthy individuals. People find it difficult to describe dissociation and may just say they felt "dizzy". The following descriptions give an indication of what sort of thing to look for:

- "I felt as if I was there, but not there, as if I was outside of myself"

- "I was spaced out, in a place all of my own"

- "Things around me didn't feel real, it was like I was watching everything on television"

- "My body didn't feel like my own"

- "I couldn't see but I could hear everyone, I just couldn't reply".

Dissociative symptoms are not diagnostic of a functional problem, but are worth looking for, particularly in patients with functional paralysis or non-epileptic attacks, because:

- they are frightening to patients who are often relieved to discover that the symptom is common and does not indicate "madness"

- where there is dissociation, there is a reasonable chance of finding that the patient has panic attacks (episodic severe anxiety)

- they can offer an extra way of explaining to patients the link between their experiences and the development of unusual symptoms such as a limb that no longer feels as if its part of them.

\section{What happened with previous doctors?}

Ask your patient to tell you about doctors who they saw previously. They may complain bitterly about Dr X or Y who "didn't listen" to them or who told them it was "nothing serious". You do not need to say whether you agree with Dr X or Y but hearing about this serves two important purposes. Firstly, it can warn you about explanations and treatments that are likely to be rejected. Secondly, by letting the patient talk openly about previous disappointing medical encounters you are showing them that you are interested in their suffering and understand their frustration.

\section{Asking about illness beliefs}

What does the patient think is causing their symptoms? What do they think should be done about them? Do they think they are irreversible or reversible? There is evidence that patients with functional neurological symptoms are more likely to be convinced that their symptoms are caused by disease than patients whose symptoms are actually caused mainly by disease-perhaps because they are trying to convince others that their symptoms are "real". These

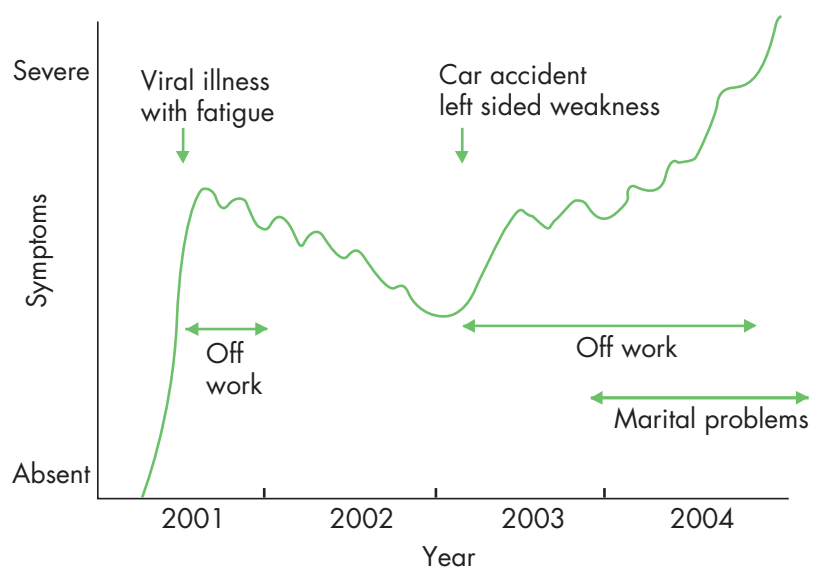

Figure 2 Using a graph to take a history from a patient with functional symptoms. 
questions also guide the final explanation. For example, if a patient expresses fear that their symptoms are due to multiple sclerosis a specific explanation of why this is not the case will be needed.

\section{Past medical history: "get the notes"}

Apart from the overall number of symptoms, the other general diagnostic red flag is whether there is a history of previous functional symptoms (table 1 ). The more functional symptoms they have had in the past, the more likely it is that the current symptom is also functional. ${ }^{2}$ This reflects the fact that some people are more prone to developing symptoms than others, for reasons we will discuss later. There may also be a history of medical attempts to treat these symptoms with surgical operations (for example, hysterectomy at a young age, appendicectomy, laparoscopy to investigate abdominal pain). Patients may have forgotten previous problems or they may just sense that the more they tell you about previous medical encounters that ended in no diagnosis, the less likely you are to take their current symptoms seriously. That is why you need the notes. Previous psychiatric diagnoses may be particularly unforthcoming in the history. If the patient already has a neurological or other disease diagnosis, ask yourself if the evidence recorded in the notes justifies it-it may not. Alternatively, they may have a disease but one which is insufficient to explain the current symptoms.

\section{Social history: work, money, the law, and marriage} An unpleasant job, being in a "benefit trap" (where money received on benefits is comparable to that earned at work), and involvement in a legal case should not be seized on as "the cause" of symptoms. This is just another form of unhelpful oversimplification. They could, however, be highly relevant obstacles to recovery. For example, patients with motor symptoms who got married or divorced have been found to do better than those patients whose marital status does not change.

\section{Modelling}

A history of similar illness in friends or family or contact with illness through work may lead to another simplistic explanation that the patient is copying or "modelling" their symptoms on others. Although plausible, there is little evidence to support or refute the idea that this occurs.

\section{Asking about emotional symptoms: go carefully}

Depression, anxiety, and panic are more common in patients with functional symptoms than those with disease. However, asking about psychological symptoms in the wrong way can make the patient defensive because they think that you are about to dismiss them as "psychiatric". We therefore suggest that you:

- make sure you have already asked about all the associated "somatic" symptoms first-for example, fatigue, poor concentration, poor sleep

- leave questions about emotions until the end of the history

- when you do ask, frame the question in terms of the symptom they are presenting with

- avoid, initially at least, psychiatric terms like depression, anxiety and panic.

For example, instead of "Have you been feeling depressed?" try "Do your symptoms ever make you feel down or frustrated?". Instead of "Do you enjoy things any more?" try "How much of the time do your symptoms stop you enjoying things?". When the patient replies that they can't enjoy things because they can't walk, etc, ask them how often they can enjoy the things they can do.

If you suspect your patient has been having panic attacks or is agoraphobic ask "Do you ever have attacks where you have lots of symptoms all at once? When do these happen? Is it when you're outside or in certain situations?".

Reading this you may ask yourself: why not just ask the patient directly about depression and anxiety? Many patients, and not just those with functional symptoms, regard anything "psychological" as mental weakness, madness, or an accusation that they are "making up" their symptoms. Being careful about how you ask questions about psychological symptoms and deferring them to later in the interview allows the patient to gain more confidence in you as a doctor. We find that once a patient trusts you are not going to use emotional symptoms "against" them they often will tell you important things they might otherwise not have done.

\section{History of abuse: to ask or not to ask?}

Childhood abuse and neglect is another factor that makes people more prone to functional symptoms. But unless you have a long time to spend with the patient or they volunteer the information, we would suggest leaving questions about early life experiences and abuse until subsequent consultations (or to someone else). The evidence from primary care currently does not support the idea that quickly "getting to the bottom of things" in this way improves outcome.

\section{How long should all this take?}

Like surgery, there is a limit to how quickly this can be done in a very complicated patient, even with the efficiencies we have suggested. Doing it in 10 minutes may be worse than not doing it all.

\section{EXAMINATION}

The diagnosis of motor and sensory symptoms discussed below depends on demonstrating positive functional signs as well as the absence of signs of disease. ${ }^{5}$ Most of these signs relate to inconsistency, either internal (for example, Hoover's sign reveals discrepancies in leg power) or external (for example, tubular field defect is inconsistent with the laws of optics).

When considering functional motor or sensory signs remember that:

- inconsistency is evidence that signs are functional, but does not tell you whether they are consciously or unconsciously produced

- the presence of a positive functional sign does not exclude the possibility that the patient also has disease-they may have both

- all physical signs have limited sensitivity, specificity, and inter-rater reliability.

\section{GENERAL SIGNS}

\section{La belle indifférence}

"La belle indifférence", an apparent lack of concern about the nature or implications of symptoms or disability, is a clinical feature that continues to receive prominence in standard descriptions of conversion disorder. However, it has no discriminatory value. Furthermore, in our experience most 
patients who are said to have "la belle indifférence" are either: (1) making an effort to appear cheerful in a conscious attempt to not be labelled as depressed; or (2) factitious (because they are deliberately making up the symptom they are not concerned about it).

The laterality of the symptoms

Although often considered left sided, a recent systematic review found only a slight left sided preponderance (55-60\%) for functional motor and sensory symptoms.

\section{FUNCTIONAL WEAKNESS \\ Preliminary observation}

Look for evidence of inconsistency. For example, compare their gait when they leave the consulting room to when they came in? What happens to their weakness when they have to take their clothes on or off or when they have to get something from their bag?

\section{Hoover's sign and other tests of "complemental opposition"}

Hoover's sign, described in 1908, is the most useful test for functional weakness and the only one that has been found in controlled studies to have good sensitivity and specificity. ${ }^{6}$ It is a simple, repeatable test, which does not require skilled or surreptitious observation. The test relies on the principle that we extend our hip when flexing our contralateral hip against resistance (you can test this out on yourself). It can be performed in two ways:

- Hip extension-Look for a discrepancy between voluntary hip extension (which is often weak) and involuntary hip extension (which should be normal) when the opposite hip is being flexed against resistance (fig 3). It is important when testing involuntary hip extension to ask the patient to concentrate hard on their good leg.

- Hip flexion-Test hip flexion in the weak leg while keeping your hand under the good heel. Look for the absence of downward pressure in the good leg.

A similar principle can be used to examine weakness of hip abduction which may initially be weak but then come back to normal if tested simultaneously with the "good side".

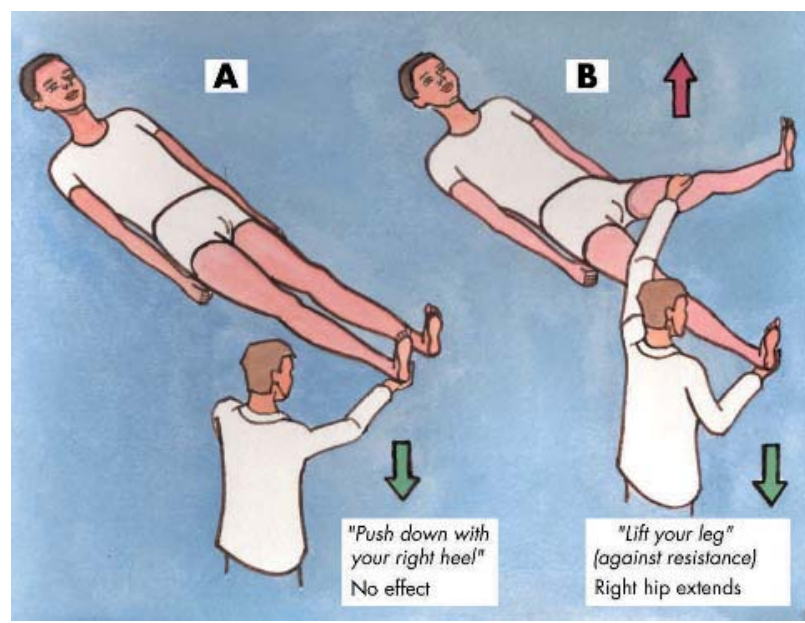

Figure 3 Hoover's sign. (A) Hip extension is weak when tested directly. (B) Hip extension is normal when the patient is asked to flex the opposite hip. Reproduced from Stone et al, ${ }^{5}$ with permission of the BMJ Publishing Group.
These tests, although useful, should be interpreted cautiously for the following reasons:

- Pain in the affected hip may produce greater weakness on direct, compared with indirect, testing as a result of attentional phenomena (related to pain rather than weakness)

- Cortical neglect can cause a positive Hoover's sign

- The test may be mildly positive in normal individuals because of a splinting effect

- None of the studies testing its utility were blinded and none mention the problem of neglect.

\section{Collapsing weakness}

"Collapsing weakness", the phenomenon in which a limb collapses from an instructed position with a light touch, is a common finding in patients with functional weakness. It is often associated with power that comes and goes or "intermittency". This should be not be described as "intermittency of effort" since you cannot directly assess someone's effort. Normal power can often be achieved transiently with encouragement, for example by saying to the patient, "At the count of three, stop me from pushing down...". Alternatively, gradually increase the force applied to the limb starting gently and building imperceptibly up to normal force.
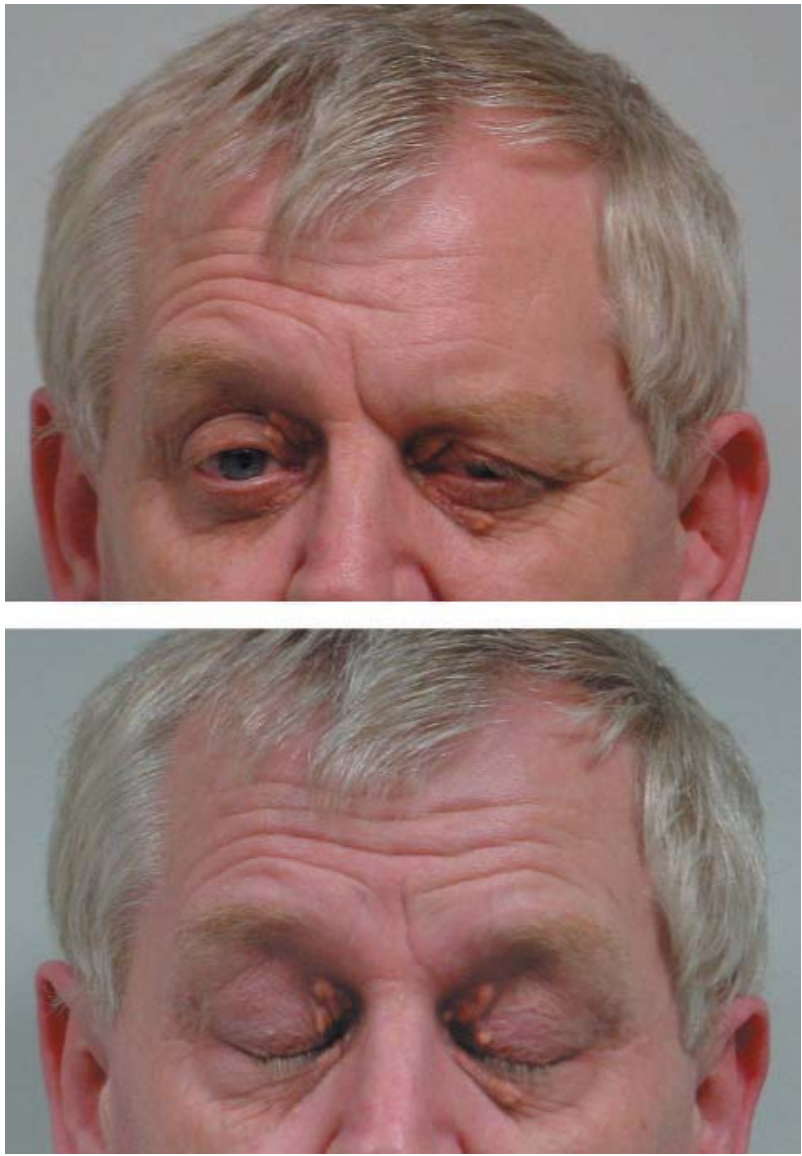

Figure 4 Pseudoptosis. This man presented with photophobia and difficulty elevating the right side of his forehead. The photograph shows his normal resting state (upper panel) and normal movement of his forehead with his eyes shut (lower panel). There is overactivity of his orbicularis oculis which had been incorrectly interpreted as ptosis. It improved with gradual exposure to light. Reproduced from Stone, ${ }^{17}$ with permission of Blackwells Publishing. 
An inability to understand the instruction, pain in the relevant joint, being generally unwell, and a misguided eagerness of some patients to "convince the doctor" may cause a false result. These concerns have been vindicated in the small number of validity studies of this sign which have found that it is a rather poor discriminator between functional and disease related symptoms.

\section{Functional weakness of the face, pseudoptosis, and "wrong way tongue deviation"}

Organic unilateral ptosis is usually associated with frontalis overactivity, whereas in pseudo-ptosis a persistently depressed eyebrow with a variable inability to elevate frontalis, overactivity of orbicularis, and photophobia is characteristic (fig 4). Apparent functional weakness of the lower half of the face and tongue deviation towards the normal rather than paretic side may occur because of overactivity of the affected side rather than underactivity.

\section{Other signs of functional weakness}

- "Co-contraction" describes the contraction of an antagonist muscle-for example, triceps, when the agonist muscle, biceps, is being tested.

- When carrying out the "arm-drop", look for an unusually slow and jerky descent of the arm from an outstretched position on to the lap (better and less aggressive than dropping the arm on to the patient's face).

- Occasionally when the "arm-drop" test is performed the arms remain inexplicably elevated, so called "pseudo waxy flexibility", a phenomenon akin to that seen under hypnosis.

- It may be worth examining the strength of the sternocleidomastoid which is rarely weak in disease but may often be weak in unilateral functional weakness.

\section{Using sedation/hypnosis}

In the altered mental state induced by sedative drugs or hypnosis, patients with functional weakness may begin to move their limbs normally again. Showing a video recording of this to the patient can be helpful in demonstrating to them the potential for reversibility.

\section{Important absent signs in functional weakness}

Although the conventional examination of tone and reflexes should be normal, pain may increase tone, anxiety can increase reflexes, and in the patient with unilateral symptoms there may be mild reflex asymmetry, particularly if there is attentional interference from the patient. Pseudoclonus can occur, with irregular and variable amplitude. The plantar response should not be upgoing, but do not be surprised if the plantar response is mute on the affected side in functional weakness, particularly if there is pronounced sensory disturbance.

\section{FUNCTIONAL SENSORY DISTURBANCE}

Functional sensory disturbance may be reported as a symptom or may be detected first by the examiner. While a number of functional sensory signs have been described, none appear to be specific and they should not therefore be used to make a diagnosis.

\section{Demarcation at the groin or shoulder}

Patients may describe sensory loss that ends where the leg or arm ends, at the shoulder or groin.

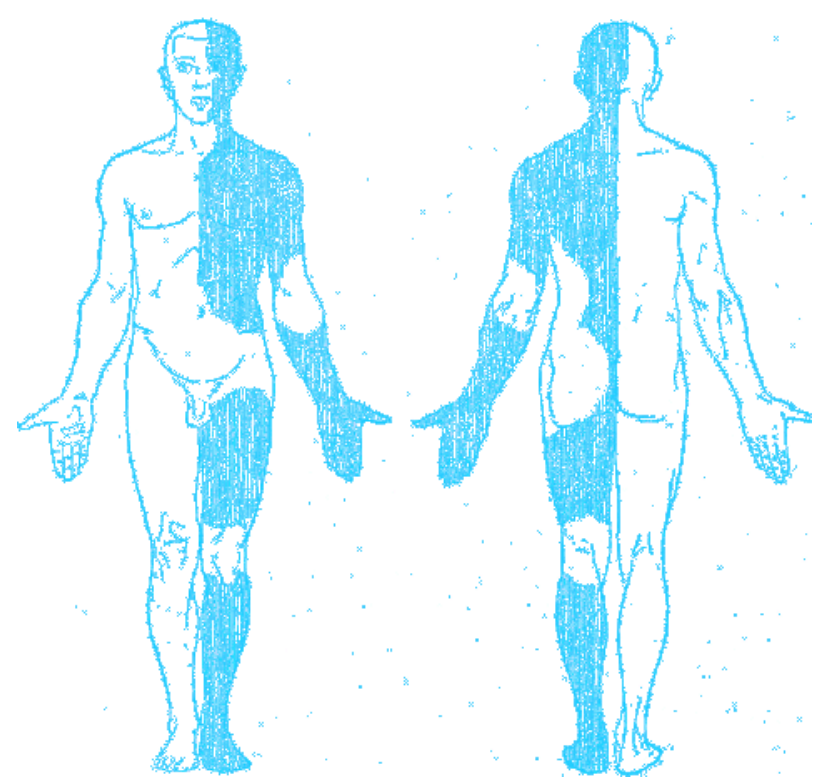

Figure 5 Hemisensory disturbance. From Charcot's clinical lectures on diseases of the nervous system, volume $3 .{ }^{18}$

\section{The "hemisensory syndrome", midline splitting, and} splitting of vibration sense

The hemisensory syndrome has been described for over a century and continues to be a well known but rarely studied clinical problem in neurology (fig 5). The intensity of the sensory disturbance often varies, and while it may be complete it is usually rather patchy, but with a distinct complaint from the patient that something is "not right" down one side or that they feel "cut in half".

Patients with hemisensory disturbance frequently complain of intermittent blurring of vision in the ipsilateral eye (asthenopia) and sometimes ipsilateral hearing problems as well. Hemisensory symptoms are increasingly recognised in patients with chronic generalised and regional pain.

"Midline splitting", the exact splitting of sensation in the midline, is said to be a functional sign because cutaneous branches of the intercostal nerves overlap from the contralateral side, so organic sensory loss should be 1 or $2 \mathrm{~cm}$ from the midline. However, midline splitting can also occur in thalamic stroke. Therefore the finding of reversible contralateral thalamic and basal ganglia hypoactivation using single photon emission computed tomography (SPECT) in patients with unilateral functional sensory symptoms is intriguing in relation to this sign. ${ }^{8}$

Similarly, patients with disease should not report a difference in the sensation of a tuning fork placed over the left compared to the right side of the sternum or frontal bone, as the bone is a single unit and must vibrate as one. Studies of both midline splitting and splitting of vibration sense have found they are common in patients with disease and so cannot be recommended. ${ }^{7}$

\section{Tests involving doctor trickery}

If you ask a patient to "Say 'Yes' when you feel me touch you and 'No' when you don't" they may indeed say "no" in the affected area. The problem in interpreting this test is firstly that the patient may be using "no" to mean "not as much", and secondly many patients will work out (at least in hindsight) that they were being tricked. This makes this test 
Table 2 Attack features that can help to distinguish non-epileptic attacks from epileptic seizures. Reproduced from Reuber and Elger ${ }^{3}$ with permission

\begin{tabular}{lll}
\hline Observation & Non-epileptic seizures & Epileptic seizures \\
\hline Situational onset & Occasional & Rare \\
Gradual onset & Common & Rare \\
Precipitated by stimuli (noise, light) & Occasional & Rare \\
Undulating motor activity & Common & Very rare \\
Asynchronous limb movements & Common & Rare \\
Purposeful movements & Occasional & Very rare \\
Rhythmic pelvic movements & Occasional & Rare \\
Opisthotonus, "arc de cercle" & Occasional & Very rare \\
Side-to-side head shaking & Common & Rare \\
Tongue biting (tip) & Occasional & Rare \\
Tongue biting (side) & Rare & Common \\
Prolonged ictal atonia & Occasional & Very rare \\
lctal crying & Occasional & Very rare \\
Closed mouth in "tonic phase" & Occasional & Very rare \\
Vocalisation during "tonic-clonic" phase & Occasional & Very rare \\
Closed eyelids & Very common & Rare \\
Convulsion $>2$ minutes & Common & Very rare \\
Resistance to eyelid opening & Common & Very rare \\
Pupillary light reflex & Usually retained & Commonly absent \\
Reactivity during "unconsciousness" & Occasional & Very rare \\
Lack of cyanosis & Common & Rare \\
Rapid postictal reorientation & Common & Rare \\
\hline
\end{tabular}

unhelpful if you want to adopt the transparent approach we favour.

\section{NON-EPILEPTIC ATTACKS}

There is a stronger evidence base for approaching the diagnosis of functional/non-epileptic attacks or pseudoseizures. $^{3}$ As for functional weakness, the history may be suggestive, but will usually not be in itself diagnostic.

\section{Semiology}

Non-epileptic attacks vary widely in their semiology but have been broadly divided into hyperkinetic/thrashing attacks and akinetic/motionless attacks. Table 2 lists some of the signs which have been tested in studies of both patients with nonepileptic attacks and epilepsy. In our experience symptoms of panic and dissociation are common in the prodromal phase, although patients may be reluctant to describe them.

As table 2 shows, there are no clinical signs of nonepileptic attacks which never occur in epilepsy, and apart from ictal electroencephalogram (EEG) abnormalities, there are no signs unique to epilepsy. For this reason, it is dangerous to use any of the listed signs in isolation to make a diagnosis.

There is a wide differential diagnosis for attacks that look "odd". "Strangeness" in itself should not lead you to a diagnosis of pseudoseizures. Frontal lobe seizures can look particularly bizarre. Paroxysmal movement disorders are another potential catch.

\section{Prolactin measurement}

Serum prolactin is often elevated 15-20 minutes after a generalised tonic-clonic seizure and should be normal after a non-epileptic attack. However, prolactin rise has been demonstrated after syncope and found to be normal after partial seizures. The test can be useful but in our experience it is often carried out badly in practice, with no baseline sample and a post-ictal specimen that is either measured too early or too late. For this reason, we do not advocate its use outside specialist units.

\section{EEG and videotelemetry}

EEG with videotelemetry remains the "gold standard" investigation for non-epileptic attacks. However, patients with partial epilepsy, particularly frontal lobe epilepsy, may not show any abnormalities on surface EEG recording when there is a deep ictal focus. In addition, some patients may not have attacks during monitoring.

\section{Using placebo and suggestion to induce attacks}

The use of intravenous placebo, such as giving a bolus of intravenous saline with the suggestion that it will bring on an attack, is controversial as it may involve deception by the doctor (depending how the procedure is explained to the patient). Verbal suggestion alone may be effective. ${ }^{9}$

\section{FUNCTIONAL OR "PSYCHOGENIC" MOVEMENT DISORDERS}

The diagnosis of a functional movement disorder is particularly challenging because of the unusual nature of some organic movement disorders. This is illustrated in the disproportionate number of movement disorders in cases where structural disease has been misdiagnosed as functional.

Further description of the features below can be found elsewhere ${ }^{4}$ and useful video material can be found accompanying a recent textbook of movement disorders. ${ }^{10}$

There are some general features common to all functional movement disorders. These include:

- Rapid onset-This is more unusual in patients with organic movement disorder.

- Variability -Variability in frequency, amplitude, or distribution may be obvious during an examination or during observation at other times. It must be remembered that all movement disorders vary to some degree and will get worse during times of stress or worry, so minor variability is not helpful.

- Improvement with distraction-Distracting tasks include asking the patient to perform tests of mental concentration (for example, serial subtraction) or physical tasks 

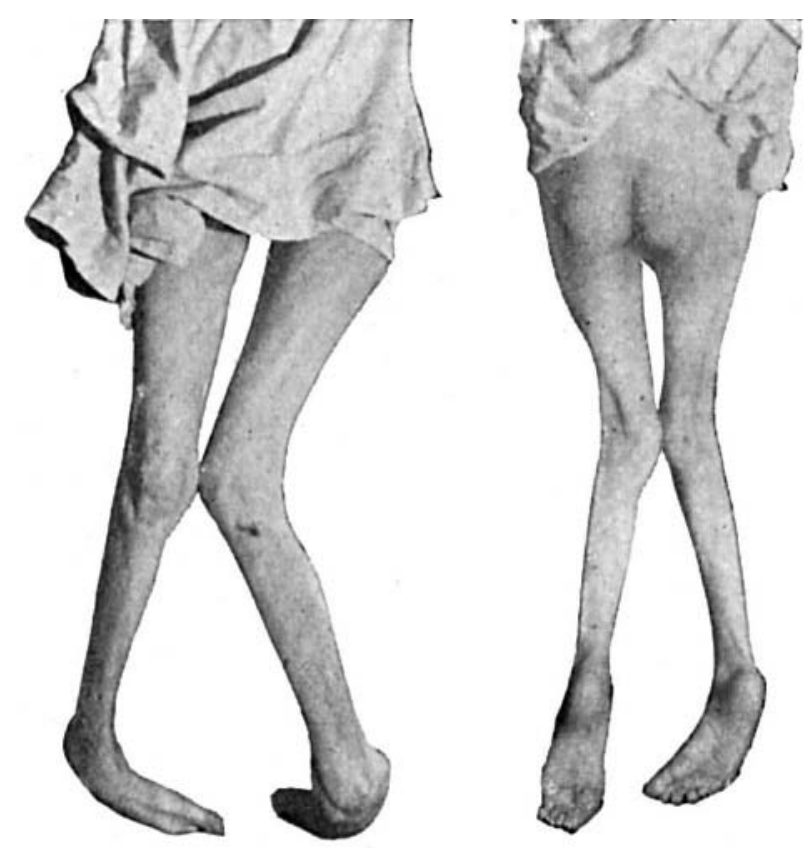

with their normal limbs (such as rapid alternating hand movements). The inverse, worsening with attention, may also occur. Again, organic movement disorders may be susceptible to these factors to a degree.

\section{Tremor}

\section{Entrainment}

When testing for entrainment, a type of distraction task, the patient is asked to make a rhythmical movement with their normal hand or foot. Either the normal limb "entrains" to the same rhythm as the abnormal side or, more commonly, the requested rhythmical movement is irregular or incomplete. There is reasonable evidence for the reliability of this test from several controlled studies. A tapping frequency of $3 \mathrm{~Hz}$ may be more discriminant, and produce more variation, than a faster $5 \mathrm{~Hz}$ rate.

Tremor amplitude change with weights/coactivation sign

When weights are added to the affected limb, patients with functional tremor tend to have greater tremor amplitude whereas in those with organic tremor the tremor amplitude tends to diminish. This may be because of coactivation of agonists and antagonist, the so-called "coactivation sign". Related to this, patients with functional tremor may shake their limb more vigorously if it is held still.

\section{Dystonia}

Patients with hysterical contracture have been described since the late 19th century alongside organic dystonia (fig 6). Psychodynamic interpretations of dystonia (such as torticollis representing a "turning away from responsibility") encouraged misdiagnosis. When this error was realised there was a backlash and the diagnosis of psychogenic dystonia almost disappeared. More recently it is being recognised again and is included in the spectrum of "fixed dystonia". ${ }^{11}$ The diagnosis is difficult but useful features include: an inverted foot or "clenched fist" onset in an adult, a fixed posture which is

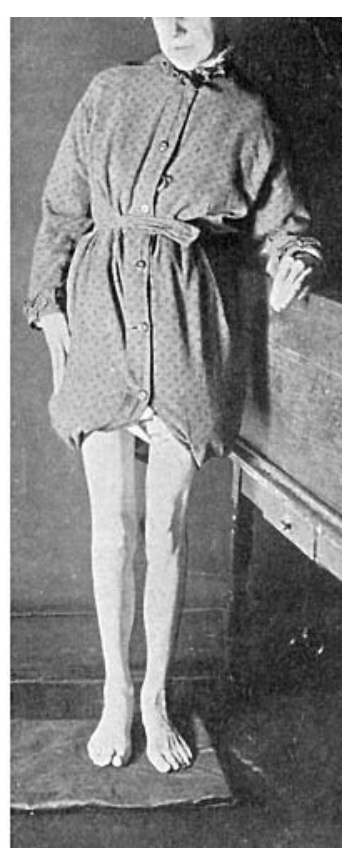

Figure 6 A patient with paraplegia and psychogenic/functional dystonia of 14 years duration before (left and middle panels) and after (right panel) treatment with psychotherapy.

Reproduced from Purves-Stewart and Worster-Drought. ${ }^{19}$

apparently present during sleep, and the presence of severe pain.

The "gold standard" for the diagnosis of functional dystonia is to demonstrate complete remission after administration of general anaesthesia, a suggestion, or placebo. Such a procedure, if handled carefully, may also be therapeutic. Be aware, however, that some types of organic dystonia may also remit spontaneously. A high proportion of patients with psychogenic dystonia have had an injury to the affected limb. There is an overlap between dystonia seen in relation to complex regional pain and psychogenic dystonia.

\section{Other movement disorders}

Psychogenic myoclonus is described as a myoclonus with variable amplitude and frequency. It may be strikingly stimulus sensitive-for example, to fluorescent lighting or with elicitation of deep tendon reflexes-in which case the latency between stimulus and jerk is often long and variable. Laborious research methods may demonstrate the presence of a "Bereitschaftspotential" one second before the jerk whereas in cortical myoclonus of organic origin there may be a cortical spike around $20 \mathrm{~ms}$ before the movement.

Psychogenic hemifacial spasm, parkinsonism, and paroxysmal movement disorders (some of which are like "partial" non-epileptic attacks) are also described. ${ }^{4}$

\section{FUNCTIONAL GAIT DISTURBANCE}

Several case series describe the features of functional gait disturbance $^{12}$ including one with video recordings. ${ }^{13}$ Variability and improvement with distraction are noted but, as with movement disorders, just because a gait looks "bizarre" or "ridiculous" does not mean it is functional.

Unilateral functional weakness of a leg, if severe, tends to produce a characteristic gait in which the leg is dragged behind the body as a single unit, like a "log" (fig 7). The hip is either held in external or internal rotation so that the foot points inwards or outwards. This may be associated with a tendency to haul the leg on to an examination couch with both hands. 

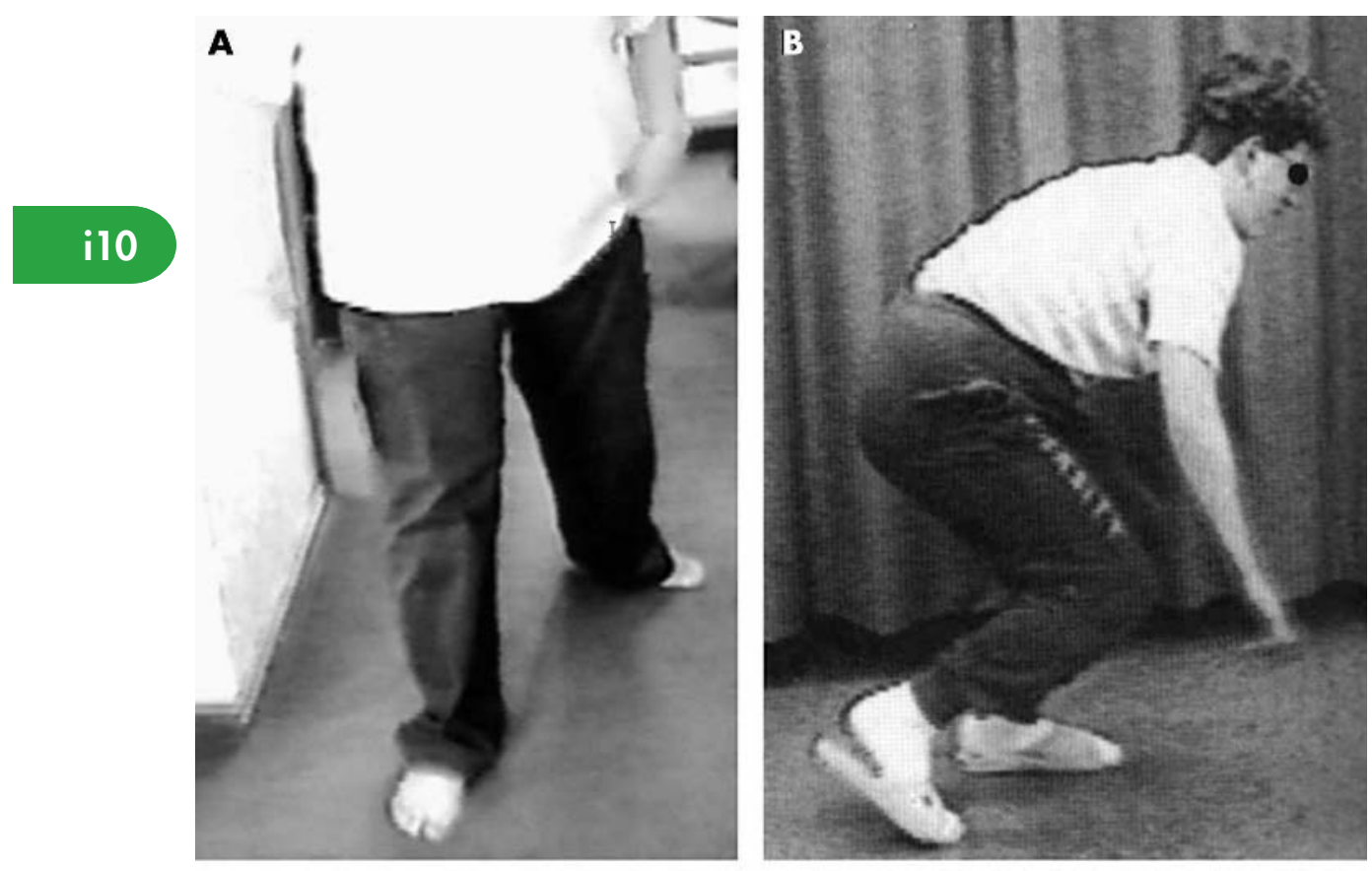

Figure 7 Functional gait disorders. (A) Dragging monoplegic gait.

(B) Uneconomic posture.

(C) Pseudoataxia. (D) "Walking on ice" gait. Fig 7A reproduced from Stone et $a l_{1}^{5}$ with permission of the BMJ

Publishing Group. Fig 7B-D reproduced from Lempert et al, ${ }_{12}^{12}$ with permission of Karger Publishing.
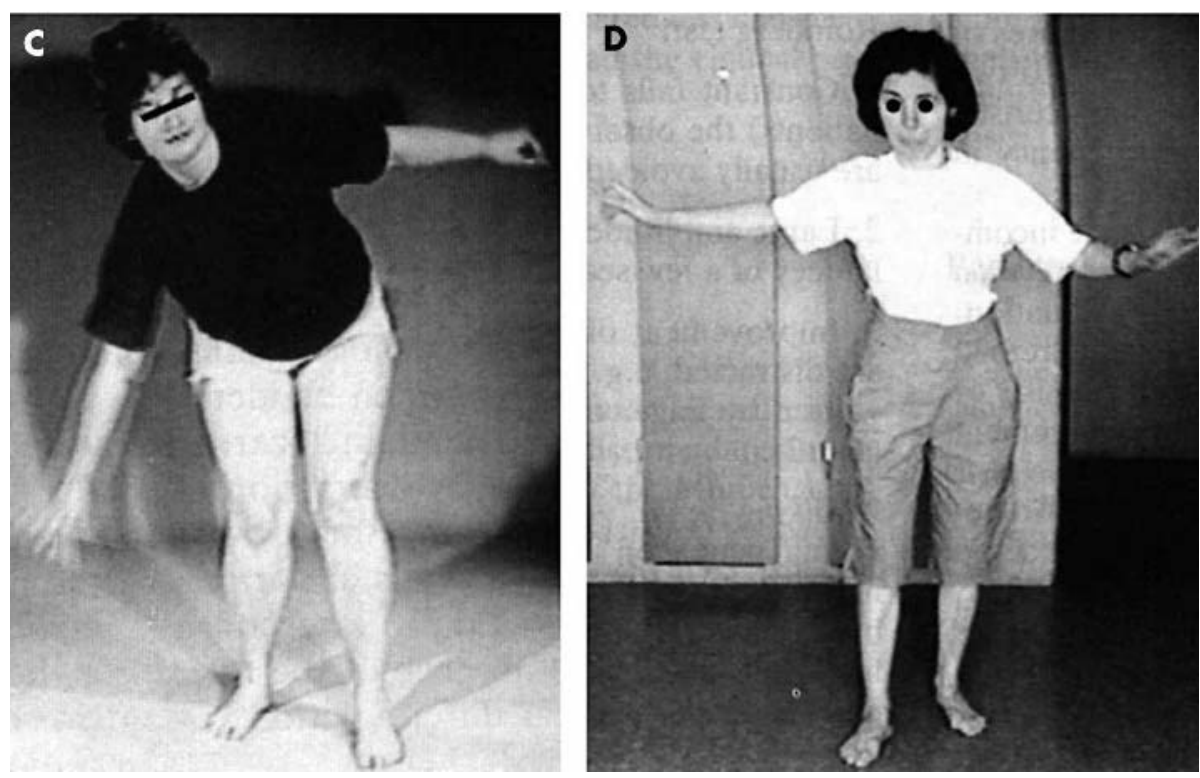

Other features suggestive of a functional gait (fig 7) include:

- Excessive slowness-Dramatic delay in gait initiation and subsequent "foot-sticking" without the subsequent improvement seen in extrapyramidal disorders.

- Falling towards or away from doctor

- "Walking on ice" pattern-The gait pattern of a normal person walking on slippery ground. Cautious, broad based steps with decreased stride length and height, stiff knees and ankles. Arms sometimes abducted as if on a tightrope.

- Uneconomic postures with waste of muscle energy-A gait with an eccentric displacement of centre of gravity such as standing and walking with flexion of hips and knees. Often associated with fear of falling.

- Sudden knee buckling-Patients usually prevent themselves from falling before they touch the ground. Knee buckling can occur in Huntington's chorea and cataplexy.
- Pseudoataxia-A gait characterised by crossed legs with or a generally unsteady gait with sudden sidesteps.

\section{OTHER SYMPTOMS}

A brief summary of other symptoms (excluding cognition, pain, and fatigue) is given here mainly in order to direct the interested reader to the relevant literature

\section{Dizziness}

A full discussion of how to determine whether dizziness is predominantly functional, and indeed whether such a distinction can be made, can be found elsewhere. ${ }^{14}$ A variety of terms have been used to describe the intersection of vestibular and psychogenic factors in dizziness including, phobic postural vertigo, "excessive awareness of normal sensation", and space and motion discomfort. Some key points are: 
- Anxiety and phobic avoidance of situations or head positions that bring on dizziness does not necessarily indicate a "psychogenic" aetiology

- On the other hand, such phobic avoidance may continue after the initial pathology has resolved

- Panic attacks presenting somatically with dizziness should be considered in the differential diagnosis of dizziness-look for a fear of embarrassment and inability to escape from situations in which it is likely to occur, such as supermarkets, as well as for other autonomic symptoms

- Physiological vestibular sensitivity to particular visual stimuli such as patterned lines or bright lights (sometimes called visual vertigo) may lead to symptoms that also come on in crowded places

- Depersonalisation and derealisation may be described by the patient as "dizziness". If this sensation is there all the time, the patient may have depersonalisation disorder (a chronic form of dissociation)

- Asking the patient to hyperventilate to see if that reproduces the symptoms might appear straightforward, but it has a high false positive rate in patients with dizziness cause by disease.

A full assessment of vestibular abnormalities, provoking stimuli and emotional symptoms can lead to tailored treatment in the form of vestibular rehabilitation and/or a cognitive behavioural approach regardless of the aetiology.

\section{Speech and swallowing symptoms}

Typically, functional dysarthria resembles a stutter or is extremely slow with long hesitations that are hard to interrupt. The speech may be telegrammatic consisting only of the main verbs and nouns in a sentence. In its extreme form the patient may become mute. Be careful though, as these types of speech disturbance can also be seen in patients with disease.

Word finding difficulty is a common symptom in anyone with significant fatigue or concentration problems and may compound a functional dysarthria. True dysphasia as a more severe functional symptom, however, is rare.

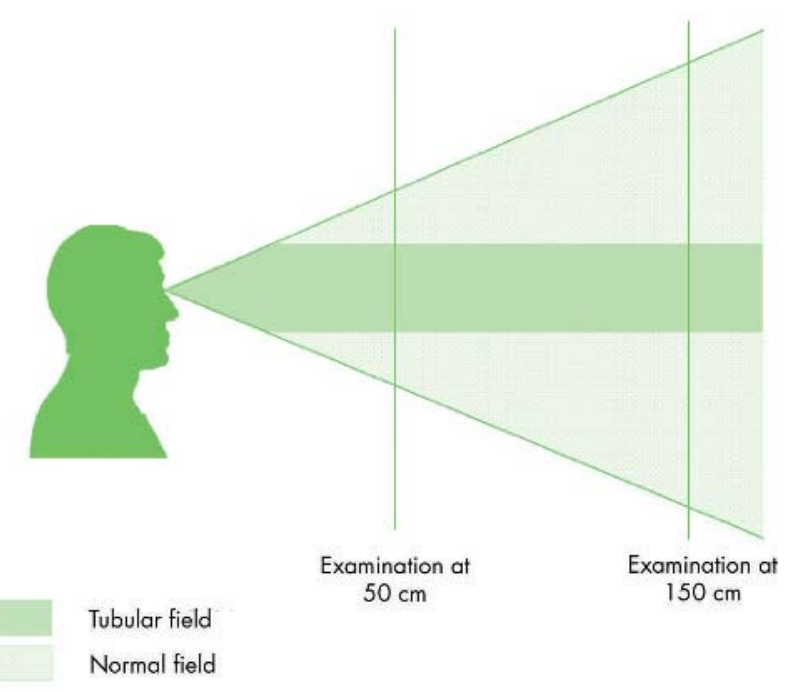

Figure 8 A "tubular" field deficit is inconsistent with the laws of optics and eye physiology. You can detect striking tubular field at the bedside.
Dysphonia is a much more common functional speech complaint and there is now quite a large literature outlining approaches to diagnosis and management. ${ }^{15}$ Often the clinical presentation is of whispering or hoarse speech that is initially thought to be laryngitis by the patient but then persists for months or years. The possibility of spasmodic adductor or abductor dysphonia must always be considered.

Globus pharyngis or functional dysphagia is common and there is also a sizeable literature about it. The patient normally complains of a sensation of a "ball in the throat" and investigations do not reveal a cause. There is controversy regarding what constitutes a full set of investigations for this symptom.

\section{Visual symptoms}

Intermittent blurring of vision that returns to normal if the patient screws up their eyes tight then relaxes them again is commonly reported. Some of these patients have convergence or accommodative spasm, with a tendency for the convergence reflex to be transiently overactive, either unilaterally or bilaterally. In this situation lateral gaze restriction can sometimes appear to be present, but the presence of miosis may help to confirm the diagnosis. Voluntary nystagmus is described and appears to be a "talent" possessed by around $10 \%$ of the population.

Tests for functional visual acuity problems are described in detail elsewhere. ${ }^{16}$ Simple bedside tests for a patient complaining of complete blindness are to ask them to sign their name or bring their fingers together in front of their eyes (which they should be able to do). They may have a normal response to menace and optokinetic nystagmus with a rotating drum (which equates to acuity of greater than $6 / 60$ ). Decreased acuity in one eye can be assessed with a "fogging test" in which "plus" lenses of increasing power are placed in front of the "good" eye until the patient can only be using their "bad" eye to see.

Spiral or tubular fields are commonly seen clinically, are often asymptomatic, and can be elicited at the bedside. Remember to test the visual fields at two distances when looking for a tubular field (fig 8). Patients with functional hemianopia have been described who have homonymous hemianopia with both eyes open and then, inconsistent with this, have a monocular hemianopia in one eye with full fields in the other eye. Monocular diplopia or polyopia may be functional but can be caused by ocular pathology.

\section{Auditory symptoms}

Basic tests for deafness rely on a startle response such as making a loud unexpected "clap" out of sight of the patient. Auditory brainstem evoked responses or evoked otoacoustic emissions may be necessary to fully investigate a patient with this symptom.

\section{INVESTIGATIONS}

Even after finding clear positive evidence of functional symptoms, investigations are necessary in many (but not all) patients. Our criteria for performing tests are either (1) we are uncertain of the diagnosis, or (2) the patient remains uncertain of the diagnosis even though we are (and have done our best to explain it to them). Some patients really do not want tests; they just want a confident opinion. Others are only interested in the opinion of the scanner. As a general rule of thumb, if you are carrying out investigations to convince or reassure the patient, remember that this may 
only be temporarily effective in patients with severe health anxiety who can become "addicted" to the reassurance of investigations. Similarly, patients who are convinced they have a certain disease like multiple sclerosis, but are not in the least anxious about this possibility, will not necessarily accept a negative investigation anyway. In a sizeable number

of patients, normal investigations will be helpful and can speed recovery.

Preferably investigations should be performed as quickly as possible, as protracted testing maintains a focus on looking for disease rather than on rehabilitation. The need to look for disease also needs to be balanced against the risk of uncovering laboratory or radiological abnormalities that have nothing to do with the symptoms but which may delay or disrupt positive management. If tests are abnormal and relevant then positive functional signs should not necessarily be ignored. It may be necessary to make two diagnoses-one of an organic disease such as multiple sclerosis and another of additional functional weakness or disability.

\section{CONCLUSIONS: ASSESSMENT AND DIAGNOSIS}

Functional symptoms are one of the most common reasons for consulting a neurologist. The assessment of such patients can be made more efficient and interesting by adapting the history, obtaining all the symptoms early on, asking about illness beliefs, and being careful about how and when you ask about psychological symptoms. In making the diagnosis the presence of positive functional signs are of key importance but should be used cautiously. Finally, be prepared to make a diagnosis of additional functional disability in someone with a known organic disorder.

\section{ACKNOWLEDGEMENTS}

We would like to thank our colleagues in Edinburgh, especially Dr Adam Zeman and Professor Charles Warlow, for their valuable collaboration and support of this work. Thanks also to Will Whitely, Rustam Al-Shahi, Malcolm McLeod, and Patrick Fox for comments on the manuscript.

\section{................}

Authors' affiliations

J Stone, A Carson, M Sharpe, NHS Department of Clinical

Neurosciences, Western General Hospital, and School of Molecular and

Clinical Medicine, University of Edinburgh, Edinburgh, UK

\section{REFERENCES}

1 Carson AJ, Ringbauer B, Stone J, et al. Do medically unexplained symptoms matter? A prospective cohort study of 300 new referrals to neurology outpatient clinics. J Neurol Neurosurg Psychiatry 2000;68:207-10.

- Providing that "one third" figure and showing that disability and distress are comparable to patients with disease.
2 Wessely S, Nimnuan C, Sharpe M. Functional somatic syndromes: one or many? Lancet 1999;354:936-9.

- A review article examining the similarities between symptom syndromes in different medical specialities.

3 Reuber M, Elger CE. Psychogenic nonepileptic seizures: a review and update. Epilepsy and Behaviour 2003;4:205-16.

- A thorough recent review of the exponentially increasing literature on non-epileptic attacks.

4 Thomas M, Jankovic J. Psychogenic movement disorders: diagnosis and management. CNS Drugs 2004; 18:437-52

- A summary of the literature on functional movement disorders.

5 Stone J, Zeman A, Sharpe M. Functional weakness and sensory disturbance. J Neurol Neurosurg Psychiatry 2002;73:241-5.

- A review of physical signs of functional weakness and sensory disturbance.

6 Ziv I, Dialdetti R, Zoldan Y, et al. Diagnosis of "non-organic" limb paresis by a novel objective motor assessment: the quantitative Hoover's test. J Neurol 1998;245:797-802.

- A neurophysiological study supporting the validity of Hoover's sign. Proof that science is possible in this area.

7 Gould R, Miller BL, Goldberg MA, et al. The validity of hysterical signs and symptoms. J Nerv Ment Dis 1986;174:593-7.

- An often quoted study showing that so called "hysterical signs" such as "la belle indifférence", collapsing weakness and midline splitting were often found in patients presenting with acute stroke.

8 Vuilleumier P, Chicherio C, Assal F, et al. Functional neuroanatomical correlates of hysterical sensorimotor loss. Brain 2001;124:1077-90.

- The most convincing functional imaging study of patients with functional motor and sensory symptoms.

9 McGonigal A, Oto M, Russell AJ, et al. Outpatient video EEG recording in the diagnosis of non-epileptic seizures: a randomised controlled trial of simple suggestion techniques. J Neurol Neurosurg Psychiatry 2002;72:549-51.

- Describing a method of inducing non-epileptic attacks during videotelemetry using simple suggestion rather than deceptive placebo.

10 Sawle G, Brown P. Movement disorders in clinical practice. Oxford: Isis Medical Media, 1999.

- Contains a CD Rom with several videos of patients with functional movement disorders including a demonstration of tremor entrainment.

11 Schrag A, Trimble M, Quinn N, et al. The syndrome of fixed dystonia: an evaluation of 103 patients. Brain 2004; 127:2360-72.

- A clinical exploration of patients with permanently clenched fists, inverted feet and the like.

12 Lempert T, Brandt T, Dieterich $M$, et al. How to identify psychogenic disorders of stance and gait. A video study in 37 patients. J Neurol 1991;238:140-6.

- A practical and detailed review of functional gait disorder with plenty of photos.

13 Hayes MW, Graham S, Heldorf P, et al. A video review of the diagnosis of psychogenic gait: appendix and commentary. Mov Disord 1999;14:914-21.

- A very helpful "video article" of 22 gaits, half of whom have functional gaits. Great teaching material as it also includes "standard" gait abnormalities.

14 Furman JM, Jacob RG. Psychiatric dizziness. Neurology 1997;48:1161-6.

- A thoughtful review of the role of psychological factors in dizziness.

15 Roy N. Functional dysphonia. Curr Opin Otolaryngol Head Neck Surg 2003;11:144-8.

- An review of functional dysphonia.

16 Beatty S. Non-organic visual loss. Postgrad Med J 1999;75:201-7.

- An review of clinical signs used in the detection of functional visual symptoms.

17 Stone J. Pseudoptosis. Practical Neurology 2002;2:364-5.

18 Charcot JM. Clinical lectures on diseases of the nervous system. London: New Sydenham Society, 1889.

19 Purves-Stewart J, Worster-Drought C. The psychoneuroses and psychoses. In: Diagnosis of nervous diseases. Baltimore: The Williams and Wilkins Company, 1952:661-758. 